\title{
Granulometric characterization of paleosols in loess series by automated static image
} analysis

György Varga ${ }^{1, *}$, János Kovács $^{2,3}$, Zoltán Szalai $^{1,4}$, Csaba Cserháti $^{5}$, Gábor Újvári ${ }^{6}$ (1)

${ }^{1}$ Geographical Institute, Research Centre for Astronomy and Earth Sciences, Hungarian Academy of Sciences, Budaörsi út 45, H-1112 Budapest, Hungary

${ }^{2}$ Department of Geology \& Meteorology, University of Pécs, Ifjúság u. 6, H-7624 Pécs, Hungary

${ }^{3}$ Environmental Analytical \& Geoanalytical Research Group, Szentágothai Research

Centre, University of Pécs, Ifjúság u. 20, H-7624 Pécs, Hungary

${ }^{4}$ Department of Environmental and Landscape Geography (Institute of Geography and Earth Sciences, Faculty of Science), Eötvös University, Pázmány Péter sétány 1/c, H-1117 Budapest, Hungary

${ }^{5}$ Department of Solid State Physics, University of Debrecen, Bem tér 18/b, H-4026 Debrecen, Hungary

${ }^{6}$ Institute for Geological and Geochemical Research, Research Centre for Astronomy and Earth Sciences, Hungarian Academy of Sciences, H-1112 Budapest, Budaörsi u. 45., Hungary

*corresponding author; e-mail: varga.gyorgy@csfk.mta.hu

\section{Abstract}


An automated image analysis method is proposed here to study the size and shape of siliciclastic sedimentary particles of paleosols of Central European loess sequences. Several direct and indirect measurement techniques are available for grain size measurements of sedimentary mineral particles. Indirect techniques involve the use of some kind of physical laws, however, all requirements for calculations are in many cases not known. Even so, the direct manual microscopic observation and measurement of large, representative number of grains is timeconsuming and sometimes rather subjective. Therefore, automated image analyses techniques provide a new and perspective way to analyse grain size and shape sedimentary particles.

Here we test these indirect and direct techniques and provide new granulometric data of paleosols. Our results demonstrate that grain size data of the mineral dust samples are strongly dependent on shape parameters of particles, and shape heterogeneity was different of the different size classes. Due to the irregular grain shape parameters, uncertainties have arisen also for the sizes.

In this paper we present a possible correction procedure to reduce the differences among the results of the laser diffraction and image analysis methods. By applying new correction factors, results of the two approaches could be get closer but the most definite factor, the unknown thickness of particles remained a problem to solve. The other presented method to assess the uncertain $3^{\text {rd }}$ dimension of particles by their intensity-size relationships makes us able to reduce further the deviations of the two sizing methods.

Keywords: image analyses, particle shape, grain size, paleosols

\section{Introduction}


Determination of granulometric parameters has been a major focus of sedimentary studies and is of growing interest in the Earth sciences (Vandenberghe et al., 2013, 2018; Újvári et al., 2016). There is a variety of instrumental techniques for the measurement of particle size. These include sieve and pipette methods through laser scattering to image analysis of pictures taken by optical or scanning electron microscopes. These various analytical methods are based on different approaches to measuring particle size. In sieving, the second largest dimension is measured as particles orientate themselves to optimally pass through the mesh, and grain size distributions are calculated from the mass of particles within different size classes (Ludwick and Henderson, 1968). Techniques based on the settling velocity of suspended particles assume that larger/heavier particles settle more rapidly from suspension than smaller/lighter ones. Particle size information of sedimentary deposits is usually determined by laser diffraction devices. This is a robust method yielding much more accurate and reliable information on grain size of windblown sediments than sieving or the gravimetrical methods (Konert and Vandenberghe, 1997; Di Stefano et al., 2010; Fisher et al., 2017; Makó et al., 2017). However, grain size data obtained with these measurements simply result from indirect estimations of sphere equivalent diameters, as calculated from the acquired laser light scattering data using mathematical transformations of different optical models (Fraunhofer and Mie theories).

Grain size characterization of irregular shaped three-dimensional sedimentary particles is a complex problem. The size of such particles is approximated by using equivalent diameters, so that the real irregular particle is replaced with an imaginary sphere or circle having similar volume, surface or area (Fisher et al., 2017). This means that sphere equivalent (SE) or circle equivalent (CE) diameters are used instead of other size parameters. However, size description of a non-spherical particle using simple indices (SE or CE diameter) consequently leads to oversimplifications. 
Not only size, but shape parameters of particles hold vital information on sedimentary transport and deposition mechanisms and post-depositional, environment-related alterations (Mazzullo et al., 1992; Pye, 1994). As the terms particle morphology, form and shape have been used in a variety of ways in published papers (Benn and Ballantyne, 1993), here, particle shape includes relative dimensions of particles, overall smoothness of particle outline and roughness. Traditional image analysis techniques have been applied widely, however previously published studies have been carried out on populations with much smaller number of particles compared to automated analyses (e.g. Dellino and La Volpe, 1996; Bagheri et al., 2015; Liu, et al., 2015). Measurement of particle shape is time-consuming (Tafesse et al., 2013). Automated static image analysis is still uncommon and underexploited for particle size and shape distribution analysis of sediments. The use of automated digital image analysis solves the issues generated by low number of measured particles as it is more precise, less time-consuming and easier to use compared with traditional methods (Baptista et al., 2012; Rodríguez et al., 2013; Campaña et al., 2016). The average particle number of automated imaging amounts to ca. $10^{4}-10^{6}$ particles, which allows us to gain statistically robust and objective insights into the morphological characteristics of particles. Various size and shape parameters, as well as optical intensity values of each particle, are routinely measured and number-size distributions can easily be converted to volumetric distributions, thus the direct comparison with results obtained by laser diffraction can be done. To date, only a few studies have been published on automated image analyses of particle size and particle shape parameters of sedimentary deposits (Rubin, 2004; Graham et al., 2005; Warrick et al., 2009; Buscombe et al., 2010), and therefore much uncertainty exists about the relationship between the different methods. Shang et al. (in press) presented grain size and shape results obtained by dynamic image analysis of Chinese loess and red clay samples. 
In this study paleosols embedded in Central European loess sequences were investigated in detail as they are the product of a complex depositional environment: granulometric characteristics of paleosols are dependent on (1) the grain size properties of the underlying windblown loess material from which the soil was formed; (2) post-depositional alteration governed by the weathering intensity characteristic for the given interstadial/interglacial period; and (3) possible syn-sedimentary dust material additions (and/or removal). However, it must be emphasized that this study is not aimed at obtaining genetically meaningful sedimentary interpretations of the samples, but instead (1) compares the grain size results obtained by widely used laser diffraction technique and by a new, high-precision granulometric characterization approach, namely automated static image analysis; (2) discusses the major differences and underlying causes; and (3) identifies problematic issues of grain size and shape determinations of the automated static image analysis technique.

Details of physicochemical environment of entrainments, transport, accumulation and postdepositional alterations of sedimentary particles can partly be reconstructed using proxies of grain size and various grain shape parameters (e.g. particle circularity, convexity, relative lengths of orthogonal axes) of sediments (Weltje and Prins, 2007; Bokhorst, et al., 2011; van Hatteren et al., in press; Schulte et al., in press; Schulte and Lehmkuhl, in press; Varga et al., in press). This is especially true for well sorted aeolian dust deposits with a fairly narrow grain size range in the silt fraction as a consequence of the selective nature of sediment transport by wind (Pye, 1987). As terrestrial wind-blown deposits are among the most important archives of past environmental changes, appropriate explanation and interpretation of proxy data is another key issue (Varga et al., in press). Various aspects of aeolian sedimentation (wind strength, source distance and transport modes, etc.) can be estimated from accurate grain size data. Huge amounts of laser diffraction grain size data have accumulated over the past decades, to make 
the comparison of new and more detailed image analysis-based granulometric information with previous researches a comprehensive discussion of methodological differences is needed.

\section{Materials and Methods}

\section{$\underline{\text { Geological setting and samples }}$}

Loess deposits cover more than half of the area of the Carpathian Basin in Central Europe (Oches and McCoy, 1995; Marković et al., 2011, 2015; Újvári et al., 2014). Previous studies revealed the complex paleoenvironmental development and depositional history of the last ca. 1 million years based on multi-proxy analyses of these excellent archives (Horváth and Bradák, 2014; Újvári et al., 2014; Marković et al., 2015). Changing climatic conditions of Pleistocene glacial-interglacial periods were imprinted in windblown dust deposition and post-sedimentary alterations of accumulated sequences. Increased dust flux of dry and cold glacials provided material for the formation of typical loess deposits. The loess formation periods were interrupted by soil development during moist and mild interglacials. While the geochemical composition of loess deposits are fairly homogeneous, climatic and environmental conditions, duration and intensity of soil forming intervals were more diverse than during glacials, leading to a geochemically and sedimentary mixed pedostratigraphy of the region (Varga, 2015). Pedogenesis during interglacials were even more complex, as we have to consider syngenetic fine-grained dust addition from external source regions (e.g., from the Sahara) to the local material during accretionary soil formation (Varga et al., 2016).

The persistent decreases in weathering intensity during interglacial intervals from the Early Pleistocene to Holocene were preserved and manifested in different types of paleosols. The 
Late and younger Middle Pleistocene loess deposits are intercalated by steppe, forest-steppe

147 and brown forest soils, while the older paleosols are reddish brown, rubified soils.

148 A generalized loess-paleosol sequence was set-up primarily based on the Paks loess section on 149 the right bank of River Danube in Hungary (N46 $38^{\prime} 25^{\prime \prime}$ E1 $18^{\circ} 52^{\prime} 36^{\prime \prime}$ ), however, paleosol 150 units of MIS-5 were missing in this well-studied site (Újvári et al., 2014), reference samples for 151 the last interglacial period were collected from the Tamási section (Southwest Hungary, Transdanubian Hills; N46 $37^{\prime} 6^{\prime \prime}$ E18 $18^{\prime}$ 32"). Nine representative samples were chosen for detailed analyses from the sampled key pedostratigrahic units representing MIS-21 up to MIS5 interglacial periods (Fig 1). The MIS-13 and MIS-15 soils were excluded from sampling and subsequent analyses because of their controversial stratigraphic position and truncated appearance (Oches and McCoy, 1995; Horváth and Bradák, 2014; Újvári et al., 2014; Varga, 2015).

\section{$\underline{\text { Samples pre-treatment and grain size measurements }}$}

All samples were chemically pre-treated before granulometric measurements by adapting the widely used procedure described by Konert and Vandenberghe (1997). Three grams of sediment were treated with $10 \mathrm{ml} \mathrm{H}_{2} \mathrm{O}_{2}(30 \%)$ and $10 \mathrm{ml} \mathrm{HCl}(10 \%)$ to oxidize organic matter and dissolve carbonates before laser diffraction measurements. Subsequently, $10 \mathrm{ml}$ of $3.6 \%$ $\mathrm{Na}_{4} \mathrm{P}_{2} \mathrm{O}_{7} \cdot 10 \mathrm{H}_{2} \mathrm{O}$ was also added to the samples, which were ultrasonicated during the analyses in order to ensure particle disaggregation. There are two main reasons for carbonate removal: (1) in loess sediments secondary calcite formation creates coatings among the particles inhibiting the dispersion of individual grains; (2) separation of detrital and authigenic, postdepositional carbonates is impossible. 
Granulometric data and Raman spectra were obtained from automatic static image analysis of Malvern Morphologi G3-ID (Malvern Instruments Ltd., UK), which is an advanced particle characterization apparatus. This device allows thousands of particle shapes to be quantified in a few hours and it has recently been used for quality control in the pharmaceutical and mining industry (Kwan et al., 1999; Ulusoy and Kursun, 2011; Schneider and Marcini, 2013; Gamble et al., 2014). Nevertheless, only a few studies have exploited image-based methods in sedimentology so far, apart from preliminary studies designed to demonstrate its potential (Altuhafi et al., 2012; Polakowski et al., 2014; Duval et al., 2015; Sochan et al., 2015; Campaña et al., 2016; Nielsen et al., 2016; Polo-Díaz et al., 2016; Becker et al., in press).

In this study, $\sim 7 \mathrm{~mm}^{3}$ of sedimentary particles were dispersed onto a flat glass slide with an instantaneous (10 ms) pulse of 4 bar compressed air and $60 \mathrm{~s}$ settling time. Particle imaging was conducted using the $20 \times$ magnification lens $\left(960 \times\right.$ magnification, 40 pixel per $\mu \mathrm{m}^{2}$ resolution) of the Morphologi G3-ID device and z-stacking was enabled (two layers above and below the focal plane, equivalent to $27.5 \mu \mathrm{m}$ in total).

Size and shape parameters of $\sim 250,000$ individual particles were automatically recorded by the software of the Mavern Morphologi G3-ID device for each sample from the captured highresolution grayscale images. The most important granulometric parameter of the image analysis based grain size measurements is the circle-equivalent (CE) diameter of the non-spherical, irregular-shaped particles. This parameter is calculated as the diameter of a circle with the same area as the projected two-dimensional particle image. The number-based grain size distribution is calculated in MATLAB (version R2016a) by classification of every particle into logarithmically-spaced size classes. Default size-bin allocation of Malvern Mastersizer was chosen to these calculations to make the comparison of image analyses and laser diffraction 
results more accurate and representative; particle size data are classified into 101 logarithmically spaced size-bins in the range between 0.01 and $3000 \mu \mathrm{m}$ (the central value of the ith size-bin $=0.0081^{\mathrm{e} 0.128 \mathrm{i}}$, where $\left.\mathrm{i}=1: 101\right)$. For transforming number-based distributions into volume-based distributions CE diameter is used for the calculation of particles volume (sphere-equivalent [SE] volume) as a weighting factor. The volume of a given size bin is specified by weighting with the total SE volume of particles classed into this size range.

Length and width are estimated from major and minor axes of the particles (Malvern Instruments Ltd., 2015). The major axis is calculated as a line through the centre of mass of the two-dimensional projected image at an orientation corresponding to the minimum rotational energy of the shape. The major axis parameter is the angle of the major axis from a horizontal line, while the minor axis passes through at a right angle to the major axis. All perimeter points of the object are projected onto the major axis (minor axis), and the longest distance between the points is the length (width) of the particle as shown in Fig 2. Other simple grain size parameters as particle area or perimeter can easily be determined using the acquired images.

Grain shape parameters provide additionally information apart from size. Aspect ratio is the ratio of width and length, while elongation is 1-aspect ratio. The circularity parameter of a particle describes the proportional relationship between the circumference of a circle equal to the object's projected area and perimeter. Convexity and solidity are determined using the convex hull (theoretical rubber band wrapped around the particle - indicated as gray area on Fig 2) of the two-dimensional images. Convexity is the ratio of perimeter of the convex hull to the particle perimeter, while solidity is the ratio of the particle and convex hull areas; these are parameters of the particle edge roughness.

Simultaneously, the mean grayscale intensity and standard deviation of particles were also measured as the bottom light (diascopic) illumination transmits through the particles. White light intensity of each pixel of particles is recorded on an 8-bit $\left(2^{8}\right)$ scale from 0 to 255 , where 
intensity value of zero is white, 255 is black. The automatically recorded dimensionless values serve as a proxy of optical properties. Mean intensity values are dependent on chemical composition, mineralogy and particle thickness, while standard deviations of intensities are controlled by the heterogeneity of particle constitution and surface morphology.

Chemical analysis was performed using the built-in Raman spectrometer of the Malvern Morphologi G3-ID. Spectra were acquired from several hundreds of targeted individual particles. These were compared with library spectra (BioRad-KnowItAll Informatics System 2017, Raman ID Expert) and correlation calculations were performed to determine the mineralogy of the targeted sedimentary grains.

Image analysis-based measurements were organized into a number-based database. All of the particles have their own identity number (ID) being the primary key in the data matrix. Each row represents one particle and columns of the table are size and shape parameters. Large numbers of measured particles ensure a statistically robust and objective insight into the granulometric characteristics of the investigated samples.

\section{Filtering out stacked particles and aggregates}

Sometimes it can be noticed that particles are not individual grains (see Fig 2d), but are clumps of particles by natural aggregation of single grains or by artificial stacking of particles during dispersion onto the glass slide. Using the appropriate shape parameters, these compound objects can be filtered out. Irregularly aggregated particles often cannot be excluded using only one parameter. This is why previous studies also applied combinations of intensity and convexity (Gamble et al., 2011); circularity and convexity (Leibreandt and Le Pennec, 2015), solidity and convexity (Liu et al., 2015) to distinguish aggregated particles. As these previous papers were dealing with microcrystalline cellulose and volcanic ash, morphologically significantly 
different material than granular particles of paleosols of aeolian dust-derived loess series, in this study, we applied a new combination of parameters to filter out stacked particles using elongation (or its complementary, the aspect ratio) and circularity thresholds together. Captured two-dimensional images of aggregated particles revealed that the perimeters of these rougher objects are larger than that of individual grains with similar CE-diameter. This observation formed the basis of application of convexity values in previous studies (Gamble et al., 2011; Leibreandt and Le Pennec, 2015; Liu et al., 2015). However, perimeters of two-dimensional projections of elongated particles can also be significantly higher than those of solid ones due to circumferential pixels, so particles with low $[<0.4]$ elongation (high $[>0.4]$ aspect ratio) and low circularity $[<0.45]$ form a class representing stacked or aggregated grains.

\section{Sufficient number of measured particles}

Experiences with automated static image analysis by Malvern Morphologi G3-ID indicate that scanning of $\sim 7 \mathrm{~mm}^{3}$ of sedimentary samples on circular, $60 \mathrm{~mm}$ diameter areas of glass slides provide shape and size parameter information on $~ 1-1.5$ million particles. Since measurements are time-consuming (average 6-hour measurement time per sample), the generated data-file sizes are large and impractical, and for cost- and energy-efficiency reasons it seems important to determine the particle number sufficient for a statistically representative granulometric characterization. The large number of acquired grain images and obtained parameter data allowed us to perform a subsampling experiment. Clusters with different numbers of randomly selected particles were sub-sampled from a total of 1 million measured grains. Every subsample clusters include the results of 100-step iterations of random particle selections.

\section{Underestimation of the finest fractions by image analysis: a theoretical approach}


272 The measured CE diameter in image analysis is calculated from the two-dimensional images of 273 particles. It is generally assumed that the instantaneous pulse of compressed air disperses the

274 sedimentary particles onto the glass slide with a consistent orientation with their largest area facing to camera. However, this is only one outcome out of infinite possible projections of a three-dimensional object. During measurements made by dynamic image analysis techniques these kinds of particle orientation problems do not distort the results since freely falling particles can rotate freely in all directions (Shang et al., in press).

To demonstrate and quantify this distortion, we modelled the deformation of two-dimensional projected areas of randomly rotated, simple, theoretical three-dimensional geometric solids (Fig 3a). Shape parameters of the solids were quantified based on the edge-ratios, where $x$ is the longest edge and $x>y>z$. Platyness $(z / y)$ and aspect ratios $(y / x)$ were chosen from 0.1 to $1(0.1$, 0.5 and 1 combinations are presented in Fig $3 \mathrm{~b}$ and Table 1), while the volume of the solids was kept constant at $1 \mu \mathrm{m}^{3}$.

The XY-plane projected areas are dependent on two major factors: (1) rotation angles $\left(\alpha_{\mathrm{x}} ; \alpha_{\mathrm{y}}\right)$; and (2) shape parameters (edge-ratios) of the objects. To determine the effect of rotation angles on projected areas, the $\alpha_{\mathrm{x}}$ and $\alpha_{\mathrm{y}}$ angles were modified from $0^{\circ}$ to $179^{\circ}$ and the projected areas were calculated for every rotation angle-pairs. The mean value of the rotation-dependent XYplane projected areas is regarded as the orientation-averaged projected area representing randomly oriented object (gray surface on Fig 3c).

The introduced $\mathrm{CE}_{\text {rot }}$ ratio is the quotient of the largest face area-based $\mathrm{CE}$ diameter (it is assumed during the image analysis that this arbitrary orientation is set) and orientation-averaged projected area-based CE diameter (the projected area of a randomly oriented particle). Larger than $1 \mathrm{CE}_{\text {rot }}$ ratio values denote that the image analysis-based measurement overestimates particle size, while ratios $<1$ imply underestimation. These $\mathrm{CE}_{\text {rot }}$ ratios were calculated for every 
possible aspect ratio-platyness combinations (Fig 4a). The displayed surface shows the level of overestimation as a function of shape parameters (orientation-averaged projected area). The higher the anisotropy of particles is, the higher the chance of overestimation of image analysisbased grain size measurement is.

Volume-based distribution curves were derived from the number-based database by weighting the individual particles with their sphere-equivalent volume, this assumption of spherical shape leads to further distortion of the results. Another correcting factor, the so-called CE/SE ratio was also introduced to reduce this inaccuracy of exchange transformation from number- to volume-based distribution functions, where SE diameters are equal for all modelled objects (as the volume of all these solids were defined as $1 \mu \mathrm{m}^{3}$ ). Similarly to $\mathrm{CE}_{\text {rot }}$ ratios, CE/SE ratios were specified for every possible aspect ratio-platyness combinations (Fig 4b), so mathematical relationships among the shape and rotation determined factors and aspect ratio-platyness values were assessed.

Aspect ratio of every single particle is known, which allowed us to get a more accurate 2dimensional representation of 3-dimensional particles, only the particle thickness need to be estimated and the $\mathrm{CE}_{\mathrm{rot}}$ and $\mathrm{CE} / \mathrm{SE}$ correction factors can be determined for every investigated particles.

\section{Assessment of the $3^{\text {rd }}$ dimension of particles: intensity based thickness assessment}

As a direct consequence of the previously discussed uncertainties, the major drawback of static automated image analysis is the unknown thickness of particles. To get an approximate estimation of the third-dimension, mean intensity values of the captured grayscale images were analysed in a completely novel way. Light transmission of sedimentary particles is influenced by thickness beyond mineral composition and colour. For this intensity based thickness 
estimation method, average intensity values for all $(n=101)$ grain size classes were determined and particles with an intensity being larger than the sum of their class intensity mean and standard deviation [ $\left[\operatorname{Int}_{\text {particle-ID } i}>\right.$ mean $\left(\operatorname{Int}_{\mathrm{GSbin}-j t h}\right)+\sigma\left(\operatorname{Int}_{\mathrm{GSbin}-j t h}\right)$, where $\operatorname{Int}_{\text {particle-ID } i}$ is the intensity of $i t h$ particles from the $j$ th size class, mean( $\left.\operatorname{Int}_{\mathrm{GSbin}-j t h}\right)$ and $\sigma\left(\operatorname{Int}_{\mathrm{GSbin}-j t h}\right)$ are the average and standard deviation of the size class $j$ ] were classified as thinner (or flatter) than average ('platy') particles (Fig. 5). Later this classification of platy and more spherical particles was used during the mathematical adjustment with different assumptions for the 3rd dimension anisotropy (z/y: normal distribution for more spherical grains; $\mathrm{z} / \mathrm{y}<0.1$ for platy)

\section{Laser diffraction}

Additional grain size measurements were done using a Malvern Mastersizer 3000 laser diffraction device with Hydro LV unit to compare the new image analysis measurements with a widely used, traditional technique. There is a huge amount of published laser diffraction grain size data, however, only some of the research papers mention the drawbacks of these technique. In the case of middle and coarse silt-sized particles, majority of light is scattered by diffraction (the diffracted light has high intensity and low angle), while smaller particles refract and absorb more efficiently resulting a low intensity and wide angle scattered light. The acquired signal is transformed by the laser device software into particle size distribution data by using the Fraunhofer or the Mie scattering theory. Fraunhofer approximation is a simplified approach and the knowledge of refractive index and absorption coefficient is not required, since it is assumed that the measured particles are relatively large (over $25-30 \mu \mathrm{m}$ - about 40 times larger than the wavelength of the laser light) and opaque. More accurate particles size data can be gained by applying the Mie theory, however, as it is a solution for Maxwell's electromagnetic field equations the knowledge of optical properties (refractive index and absorption coefficient 
[imaginary part of the complex refractive index]) of the sample and the dispersant is needed.

Due to these reasons, Mie optical model provide more accurate data on the amount of smaller particles (clay and fine silt). As the knowledge of mineralogy-related optical properties is a mandatory for scattered light data to particle size Mie-transformations, bulk mineralogical composition of sediments was estimated from XRD data.

Previous XRD measurements of aeolian dust deposits in the Carpathian Basin indicated that quartz ( 30-60\%), $10 \AA$ phases (illite \pm muscovite \pm biotite: $20-30 \%$ in loess and $10-20 \%$ in paleosol), carbonates and $14 \AA$ phases (smectite \pm vermiculite \pm chlorite) were the dominant (Nemecz et al., 2000; Újvári et al., 2014). Bulk mineral composition data was used to assess the optimal optical settings of laser diffraction measurements to calculate grain size distributions by using the mineralogy-dependent complex refractive index: $1.54-0.1 \mathrm{i}$ for the sedimentary samples and $1.33 \mathrm{Ri}$ for the dispersant water (Özer et al., 2010). However, due to the polymineral composition and dependence of absorption coefficient on particle shape and surface roughness, some additional calculations were made with the combination of various refractive indices (Ri: 1.45-1.6) and absorption coefficients (Ac: 0.01-1).

\section{Scanning electron microscopy}

Hitachi S-4300 CFE Scanning Electron Microscope (SEM) micrographs were taken to document and illustrate the shape and size variability of grains. SEM uses a focused beam of electrons to create magnified images being both high contrast and extremely sharp, and therefore suitable for particle surface morphology characterization. Previous studies reported that size and shape of individual particles can be accurately assessed by image analysis software and it is considered as a direct and absolute measure of particle size (Francus, 1998; Fandrich et al., 2007). In this paper, several tens of mineral particles per sample were pictured (with 
magnification from $400 \times$ to $2000 \times$ ) by SEM to confirm the notable irregular shape and anisotropy of $3^{\text {rd }}$ dimension (thickness) of some particles.

\section{Results}

Image analysis

The acquired images of an average of 250,000 mineral particles per samples allowed us to calculate robust number- and volume-weighted size and shape distribution curves. Here, the grain size and intensity distributions are presented as both number and volume-based distributions, while other shape parameters are reported only as volume-weighted due to the of low-resolution of acquired images in the submicron fraction ( $<40$ pixel) affecting the exact determination of particle perimeter (Fig 6 and Table 2).

Size and shape parameters of samples as well as their intensity values exhibit pretty similar general characteristics for the bulk, full grain size spectrums. The number size distributions have a general bimodal nature with a pronounced submicron peak and an additional one between 8 and $10 \mu \mathrm{m}$ (Fig 6a). By contrast, the volume based CE diameter distributions are characterized with unimodal curves (closely log-normal distributions) with coarse silt-sized modal diameter values $(40-60 \mu \mathrm{m})($ Fig $6 \mathrm{~b})$.

As a logical consequence of number-based approach, most of the particles fall into the submicron fractions with high grayscale intensity values (due to their opacity) as it is reflected by the remarkable peak of the number-based intensity curve around the adjusted grayscale threshold of 144, what was selected to distinguish background from the mineral particles (Fig 6c). Applying the volume-transformations by weighting the particles with their SE volume, the modal values were found in the darker range of grayscale intensity of 50 to 80 (Fig 6d). 
General patterns of circularity and convexity distributions are resembling, both of these curves have a slight positive skewness (circularity: 1.2-2.3; convexity: 1.4-2.4) and modal values between 0.6 and 0.7 with tails extending towards more circular and convex shape directions (Fig 6e,f). Solidity of the mineral grains exhibits a rather homogeneous character with a clear positive skewness (3.1-4.5) and fairly high (>0.95) modus (Fig 6g). Aspect ratios, being the ratios of width and length values, range dominantly between 0.7 and 0.9 (Fig 6h).

Granulometric parameters of selected size fractions were also analysed. Size and shape properties of clay $(<2.00 \mu \mathrm{m})$, fine $(2.00-6.25 \mu \mathrm{m})$, medium $(6.25-20.00 \mu \mathrm{m})$ and coarse silt $(20.00-62.50 \mu \mathrm{m})$ as well as of sand (larger than $62.5 \mu \mathrm{m})$ size particle classes were separately determined. A general granulometric heterogeneity was identified towards larger size fractions, so larger particles have a more irregular shape character than the finer ones. This heterogeneity is especially well expressed for circularity and convexity with mean values decreasing from 0.95-0.97 to $0.64-0.71$ from the clay to sand fractions. Similar, but not so obvious trends could be observed for the aspect ratio and solidity parameters. However, the aspect ratio values were fairly low even for the clay-sized grains ( 0.78-0.8), translating to a $20-25 \%$ difference between particle length and width (Fig 7; Table 3).

Structural fingerprint analyses by Raman spectrometry aided mineral identifications. Due to the relatively low number of interpretable spectra, special focus was given to the main components of the samples studied (30-120 $\mu \mathrm{m}$ quartz and feldspar grains). Size and shape parameters of these particle-clusters displayed similar main characteristics. All of the previously introduced parameters were found to be almost identical, only the mean intensities of quartz grains were biased towards lighter values compared to feldspars (Table 4).

Irregularity and heterogeneous shapes of sedimentary particles could undoubtedly be observed on the obtained SEM micrographs of bulk samples (Fig 8.). Acquired images also revealed several fracture faces, V-shaped percussion marks, linear steps, and conchoidal crushing 
421 features on the grain surfaces. [According to Pye and Sperling (1983), Liu et al. (1985), Pye 422 (1995), Lu et al. (2001) and Wright et al. (2011) this kind of morphological properties of silt423 sized mineral grains are only characteristics of aeolian dust particles. Such microtextures together with the macroscopic characteristics of the silt classes indicate that these particles were primarily transported and deposited by wind, post-depositional alterations formed soils from this parent material.] The presence of fine-grained platy particles with significant $3^{\text {rd }}$ dimensional anisotropy due to their thinness was also confirmed. However, the quantification of this anisotropy proved to be impossible using these images. Nevertheless, it is clear that thickness/width ratios are by orders of magnitude smaller than width/length ratios of some particles.

\section{Laser diffraction}

Laser diffraction grain size measurements resulted in silt dominated, positively skewed (asymmetry towards the coarse fractions), unimodal distribution curves with minor, yet remarkable contribution of clay and fine-sand particles. The fine-grained tail into the direction of clay and fine silt fractions, beside the prominent maximum of medium- and coarse-silt components, is typical for aeolian dust deposits and paleosols intercalated in loess sequences. By using different complex refractive index values for grain size distribution measurements, the coarse silt-sized primary modes were not modified, however significant changes could be identified in the volumetric amount of clay and fine silt fractions (Fig 9).

\section{Discussion}

\section{Sufficient number of measured particles}


447 It was found that, depending on the parameter itself, different particle numbers provide different representations of a sediment sample (Fig 10). For volume-based CE diameter distributions, the analyses of more than 50,000 particles are required to reach $\mathrm{R}^{2}=0.9$ between the total sample and the subpopulation (Fig 10a). However, since there is a cubic relationship between particle diameter and volume, even a small number of large (coarse silt and sand) particles is able to significantly modify the coarse grained tail of the grain size distribution. This apparent modification of the distribution curve cannot be easily quantified due to the logarithmic allocation of grain size bins. To get a more robust representation of grain size of polydisperse samples (particle sizes covering several orders of magnitude, e.g., submicron to some few hundred microns of aeolian dust deposits), several millions of scanned mineral particles would be necessary. At the same time, intensity or some shape parameters can be assessed fairly well using the results of only a few thousand particles (Fig 10b,c).

\section{Underestimation of the finest fractions by image analysis: a theoretical approach}

Image analysis grain size results indicated underestimation of clay and fine silt fractions compared to laser diffraction measurements, while the modal values of the coarse silt (or fine sand) fraction were found to be higher than those obtained by laser particle sizing.

By using $\mathrm{CE}_{\text {rot }}$ ratio and $\mathrm{CE} / \mathrm{SE}$ corrections, the image analysis curves can be translated by a vector parallel to the $\mathrm{x}$-axis by $10-15 \%$ assuming a normal distribution of thickness values.

Based on the SEM images and general character of clay-minerals, this latter assumption of normally distributed thickness values brings an obvious source of error into this correction process. By extending the modelling process towards thinner particles (with z/y ratios 0.01$0.09)$, the $\mathrm{CE}_{\text {rot }}$ ratio could result in more than a $50 \%$ correction on platy particle sizes. 
472 Combined application of modelled correction factors and intensity based thickness assessment

473

474

475

476

477

478

479

Grain size and total volume of platy (more anisotropic) grains can be regarded as significantly overestimated as demonstrated by the previously deduced $\mathrm{CE}_{\text {rot }}$ (rotation averaged) ratio and $\mathrm{CE} / \mathrm{SE}$ correction factor. The flatter than average particles were classified originally into larger grain size bins which therefore have an overestimated volume. Comparison of volumetric amount of bulk samples and particles classified based on intensities as 'platy' and 'spherical' are shown in Fig 11. The introduced correction factors, even with the assumption of a normal distribution of particle thicknesses, are capable of making the CE diameters better converged, but unable to explain the larger size values themselves. The volumetric amount of more platy particles (especially clay minerals) is the most uncertain factor in these calculations, as a consequence of their significantly higher $3^{\text {rd }}$ dimension anisotropy compared to the quartz and feldspar grains.

By applying the $\mathrm{CE}_{\text {rot }}$ ratio and $\mathrm{CE} / \mathrm{SE}$ correction factor adjustment for the platy and spherical particles with different assumptions for the $3^{\text {rd }}$ dimension anisotropy (z/y: normal distribution for spherical grains; <0.1 for platy), the results of laser diffraction and image analysis measurements are in better agreement, i.e., their correlation coefficients are higher compared to the original, mathematically "untreated" results.

\section{Conclusions}

Granulometric investigations of Pleistocene interglacial paleosols intercalated into loess sequences in the Carpathian Basin revealed the major discrepancies in results obtained by the two different measurement techniques applied. The data acquired by widely used, indirect laser 
diffraction and direct observations by automated image analysis provided complementary, but different information on grain size. While the particle size distributions provided by laser diffraction measurements are dependent on the complex refractive index of a given particle (which can only be approximated in case of polymineral samples) assuming a spherical shape, the image analysis techniques are based simply on the direct, optically-acquired images of grains.

Comparisons of measured grain sizes indicated that the fine populations are consistently and significantly underestimated by the image analysis technique compared to laser scattering results. Modelling data demonstrate that the anisotropic character of irregular particles, especially the thickness of platy minerals, are responsible for the observed disagreements. The acquired two-dimensional images of dispersed particles sitting with their largest area on the glass slide were classified into grain size bins being too large based on their circle-equivalent diameter. In addition, their volumetric-weighting scores (sphere-equivalent volume derived from the $\mathrm{CE}$ diameter) were also found to be too high in volume-based conversions. Consequently, this led to overestimation of particle sizes and volumetric amounts of wrongly classified platy grains due to the cubic relationship. Application of the rotation averaged and $\mathrm{SE} / \mathrm{CE}$ ratios as correction factors successfully reduces the discrepancies between results obtained by the two approaches. Nevertheless, the most definite factor, the unknown thickness of particles still remains an unresolved problem. The other presented innovative way of estimating the uncertain $3^{\text {rd }}$ dimension of particles using their intensity-size relationships allows us to further minimize deviations between the two particle sizing methods.

However, since particle sizes of paleosols covering several orders of magnitude, even a small number of coarse grains can modify significantly the grain size distribution curves in the larger fractions distorting the whole measurement spectrum, and so the full agreement between laser diffraction and image analysis results cannot be reached. 

understanding of physical background of them. Optical dependence of laser diffraction 523 measurements should be investigated in the future, while the thickness-related uncertainties of 524 granulometric data of various deposits. image analysis must also be studied by further studies. All in all, there are uncertainties connected to both approaches, however, these two methods can be important complements of each other, providing a useful tool to decipher valuable sedimentary information from

\section{Acknowledgement}

530

\section{Support of the National Research, Development and Innovation Office NKFIH K120620 (for} G. Varga) and K120213 (for J. Kovács) are gratefully acknowledged. It was additionally supported (for G. Varga) by the Bolyai János Research Scholarship of the Hungarian Academy of Sciences.

\section{References}

Altuhafi, F., O'Sullivan, C., Cavarretta, I., 2013. Analysis of an image-based method to quantify the size and shape of sand particles. Journal of Geotechnical and Geoenvironmental Engineering 139, 1290-1307.

Bagheri, G.H., Bonadonna, C., Manzella, I., Vonlanthen, P., 2015. On the characterization of size and shape of irregular particles. Powder Technology, 141-153.

Baptista, P., Cunha, T.R., Gama, C., Bernardes, C., 2012. A new and practical method to obtain grain size measurements in sandy shores based on digital image acquisition and processing. Sedimentary Geology 282, 294-306. 
Becker, L.W.M., Hjelstuen, B.O., Støren, E.W.N., Sejrup, H.P., in press. Automated counting of sand-sized particles in marine records. Sedimentology.

Benn, D.I., Ballantyne, C.K., 1993. The description and representation of particle shape. Earth Surface Processes and Landforms 18, 665-672.

Buscombe, D., Rubin, D.M., Warrick, J.A., 2010. A universal approximation of grain size from images of noncohesive sediment. Journal of Geophysical Research: Earth Surface 115, F02015.

Campaña, I., Benito-Calvo, A., Pérez-González, A., Bermúdez de Castro, J.M., Carbonell, E., 2016. Assessing automated image analysis of sand grain shape to identify sedimentary facies, Gran Dolina archaeological site (Burgos, Spain). Sedimentary Geology 346, 72-83.

Dellino, P., La Volpe, L., 1996. Image processing analysis in reconstructing fragmentation and transportation mechanisms of pyroclastic deposits. The case of Monte Pilato-Rocche Rosse eruptions, Lipari (Aeolian islands, Italy). Journal of Volcanology and Geothermal Research 71, $13-29$.

Di Stefano C, Ferro V, Mirabile S., 2010. Comparison between grain-size analyses using laser diffraction and sedimentation methods. Biosystems Engineering 106, 205-215.

Duval, M., Campana, I., Guilarte, V., Miguens, L., Iglesias, J., Sierra, S.G., 2015. Assessing the uncertainty on particle size and shape: Implications for ESR and OSL dating of quartz and feldspar grains. Radiation Measurements 81, 116-122.

Fandrich, R., Gu, Y., Burrows, D., Moeller, K., 2007. Modern SEM-based mineral liberation analysis. International Journal of Mineral Processing 84, 310-320.

Fisher, P., Aumann, C., Chia, K., O'Halloran, N., Chandra, S., 2017. Adequacy of laser diffraction for soil particle size analysis. PLoS ONE 12, 0176510

Francus, P., 1998. An image-analysis technique to measure grain-size variation in thin sections of soft clastic sediments. Sedimentary Geology 121, 289-298. 
Gamble, J.F., Chiu, W.S., Tobyn, M., 2011. Investigation into the impact of sub-populations of agglomerates on the particle size distribution and flow properties of conventional microcrystalline cellulose grades. Pharmaceutical Development and Technology 16, 542-548. Gamble, J.F., Ferreira, A.P., Tobyn, M., DiMemmo, L., Martin, K., Mathias, N., Schild, R., Vig, B., Baumann, J.M., Parks, S., Ashton, M., 2014. Application of imaging based tools for the characterisation of hollow spray dried amorphous dispersion particles. International Journal of Pharmaceutics 465, 210-217.

Graham, D.J., Rice, S.P., Reid, I., 2005. A transferable method for the automated grain sizing of river gravels. Water Resources Research 41, 1-12.

Konert, M., Vandenberghe, J., 1997. Comparison of laser grain size analysis with pipette and sieve analysis: A solution for the underestimation of the clay fraction. Sedimentology 44, 523535.

Kwan, A.K.H., Mora, C.F., Chan, H.C., 1999. Particle shape analysis of coarse aggregate using digital image processing. Cement and Concrete Research 29, 1403-1410.

Leibrandt, S., Le Pennec, J.L., 2015. Towards fast and routine analyses of volcanic ash morphometry for eruption surveillance applications. Journal of Volcanology and Geothermal Research 297, 11-27.

Lisiecki, L., Raymo, M.E., 2005. A Pliocene-Pleistocene stack of 57 globally distributed benthic $\delta 180$ records. Paleoceanography 20, PA1003. DOI: 10.1029/2004PA001071

Liu, E.J., Cashman, K.V., Rust, A.C., 2015. Optimising shape analysis to quantify volcanic ash morphology. GeoResJ 8, 14-30.

Lu, H., Vandenberghe, J., 2001. Aeolian origin and palaeoclimatic implications of the 'Red Clay' (north China) as evidenced by grain-size distribution. Journal of Quaternary Science 16, 89-97. 
600

601

602

603

604

605

606

607

608

609

610

611

612

613

614

615

616

617

618

Ludwick, J.C., Henderson, P.L. 1968. Particle shape and inference of size from sieving. Sedimentology 11, 197-235.

Makó, A., Tóth, G., Weynants, M., Rajkai, K., Hermann, T., Tóth, B., 2017. Pedotransfer functions for converting laser diffraction particle-size data to conventional values. European Journal of Soil Science 68, 769-782.

Malvern Instruments Ltd., 2015. Morphologi G3 User Manual. Malvern, United Kingdom, 268 p.

Mazzullo, J. M., Alexander, A., Tieh, T., Menglin, D., 1992. The effects of wind transport on the shapes of quartz silt grains. Journal of Sedimentary Petrology 62, 961-971.

Nemecz, E., Pécsi, M., Hartyáni, Z., Horváth, T., 2000. The origin of the silt size quartz grains and minerals in loess. Quaternary International 67-71, 199-208.

Nielsen, P.R., Dahl, S.O., Jansen, H.L., Støren, E.N., 2016. Holocene aeolian sedimentation and episodic mass-wasting events recorded in lacustrine sediments on Langøya in Vesterålen, northern Norway. Quaternary Science Reviews 148, 146-162.

Oches, E.A., McCoy, W.D., 1995. Aminostratigraphic evaluation of conflicting age estimates for the "young loess" of Hungary. Quaternary Research 44, 160-170.

Özer, M., Orhan, M., Işik, N.S., 2010. Effect of particle optical properties on size distribution of soils obtained by laser diffraction. Environmental and Engineering Geoscience. 16,163-73. Polakowski, C., Sochan, A., Bieganowski, A., Ryzak, M., Földényi, R., Tóth, J., 2014. Influence of the sand particle shape on particle size distribution measured by laser diffraction method. International Agrophysics 28, 195-200.

Polo-Díaz, A., Benito-Calvo, A., Martínez-Moreno, J., Mora Torcal, R., 2016. Formation processes and stratigraphic integrity of the Middle-to-Upper Palaeolithic sequence at Cova Gran de Santa Linya (Southeastern Prepyrenees of Lleida, Iberian Peninsula). Quaternary International 417, 16-38. 
Pye, K.H., Sperling, C.H.B., 1983. Experimental investigation of silt formation by static breakage processes: the effect of temperature, moisture and salt on quartz dune sand and granitic regolith. Sedimentology 30, 49-62.

Pye, K., 1987. Aeolian Dust and Dust Deposits. Academic Press, London 334 p.

Pye, K., 1994. Shape sorting during wind transport of quartz silt grains - discussion. Journal of Sedimentary Research A 64, 704-705.

Pye, K., 1995. The nature, origin and accumulation of loess. Quaternary Science Reviews 14, 653-667.

Rodríguez, J.M., Edeskär, T., Knutsson, S., 2013. Particle shape quantities and measurement techniques-A review. Electronic Journal of Geotechnical Engineering 18 A, 169-198.

Rubin, D.M., 2004. A simple autocorrelation algorithm for determining grain size from digital images of sediment. Journal of Sedimentary Research 74, 160-165.

Schneider, A., Marcini, A., 2013. Morphologi G3-ID - A new instrument for problems in cement research. Cement International 11, 69-77.

Schulte, P., Lehmkuhl, F., in press. The difference of two laser diffraction patterns as an indicator for post-depositional grain size reduction in loess-paleosol sequences. Palaeogeography, Palaeoclimatology, Palaeoecology

Schulte, P., Sprafke, T, Rodrigues, L., Fitzsimmons, K.E., in press. Are fixed grain size ratios useful proxies for loess sedimentation dynamics? Experiences from Remizovka, Kazakhstan. Aeolian Research

Shang, Y., Kaakinen, A., Beets, C.J., Prins, M.A., in press. Aeolian silt transport processes as fingerprinted by dynamic image analysis of the grain size and shape characteristics of Chinese loess and Red Clay deposits. Sedimentary Geology. 
642 Sochan, A., Zieliński, P., Bieganowski, A., 2015. Selection of shape parameters that 643 differentiate sand grains, based on the automatic analysis of two-dimensional images. 644 Sedimentary Geology 327, 14-20.

645 Tafesse, S., Robison Fernlund, J.M., Sun, W., Bergholm, F., 2013. Evaluation of image analysis 646 methods used for quantification of particle angularity. Sedimentology 60, 1100-1110.

647 Újvári, G., Varga, A., Raucsik, B., Kovács, J., 2014. The Paks loess-paleosol sequence: A 648 record of chemical weathering and provenance for the last 800ka in the mid-Carpathian Basin. 649 Quaternary International 319, 22-37.

650 Újvári, G., Kok, J.F., Varga, G., Kovács, J., 2016. The physics of wind-blown loess: 651 Implications for grain size proxy interpretations in Quaternary paleoclimate studies. Earth652 Science Reviews 154, 247-278.

653 Ulusoy, U., Kursun, I., 2011. Comparison of different 2D image analysis measurement 654 techniques for the shape of talc particles produced by different media milling. Minerals 655 Engineering 24, 91-97.

656 van Hateren, J.A., Prins, M.A., van Balen, R.T., in press. On the genetically meaningful 657 decomposition of grain-size distributions: A comparison of different end-member modelling 658 algorithms. Sedimentary Geology

659 Vandenberghe, J., 2013. Grain size of fine-grained windblown sediment: A powerful proxy for 660 process identification. Earth-Science Reviews 121, 18-30.

661 Varga, G., 2015. Changing nature of pleistocene interglacials-is it recorded by paleosoils in 662 hungary (Central Europe)? Hungarian Geographical Bulletin 64, 313-322.

663 Varga, G, Cserháti, Cs., Kovács, J., Szalai, Z., 2016. Saharan dust deposition in the Carpathian 664 Basin and its possible effects on interglacial soil formation. Aeolian Research 22, 1-12. 
665 Varga, Gy., Újvári, G., Kovács, J., in press. Interpretation of sedimentary (sub)populations 666 extracted from grain size distributions of Central European loess-paleosol series. Quaternary 667 International

668 Warrick, J.A., Rubin, D.M., Ruggiero, P., Harney, J.N., Draut, A.E., Buscombe, D., 2009. 669 Cobble cam: Grain-size measurements of sand to boulder from digital photographs and 670 autocorrelation analyses. Earth Surface Processes and Landforms 34, 1811-1821.

671 Wright, J.S., 2001. Desert loess versus glacial loess: Quartz silt formation, source areas and 672 sediment pathways in the formation of loess deposits. Geomorphology 36, 231-256.

673

674

675 

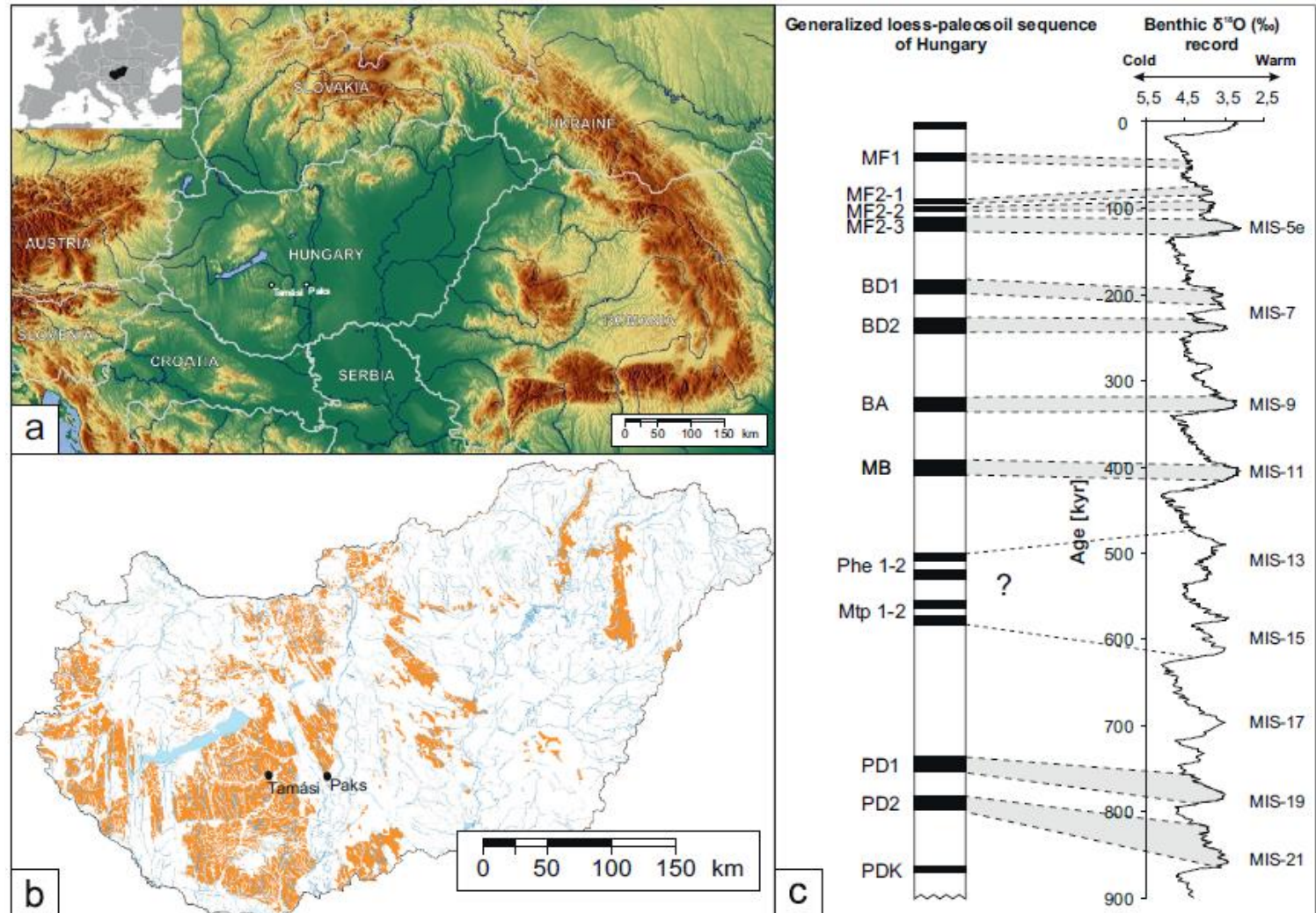

678 Figure 1. Origin of samples: (a) location map of investigated sites in the Carpathian Basin; (b)

679 loess distribution map of Hungary; (c) generalized loess-paleosol sequence of Hungary and its 680 possible correlation with benthic $\delta^{18} \mathrm{O}$ record of deep sea sediments (Lisiecki and Raymo, $6812005)$. 

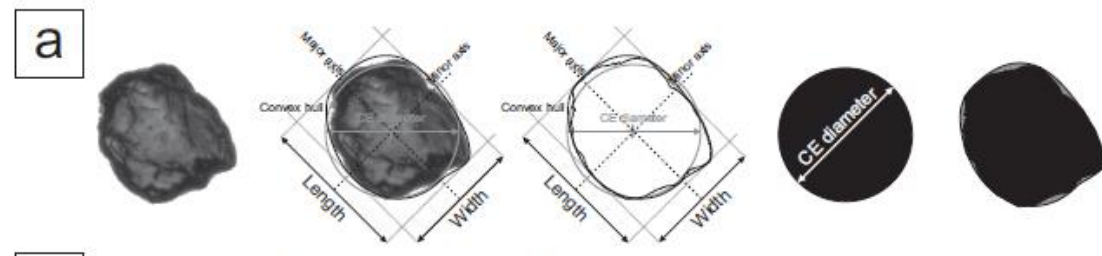

$\mathrm{b}$
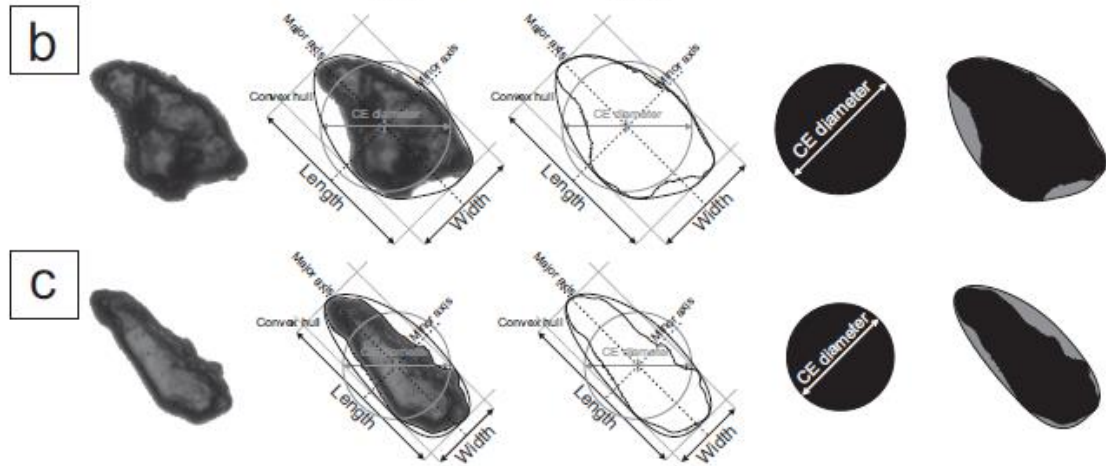

\section{d}
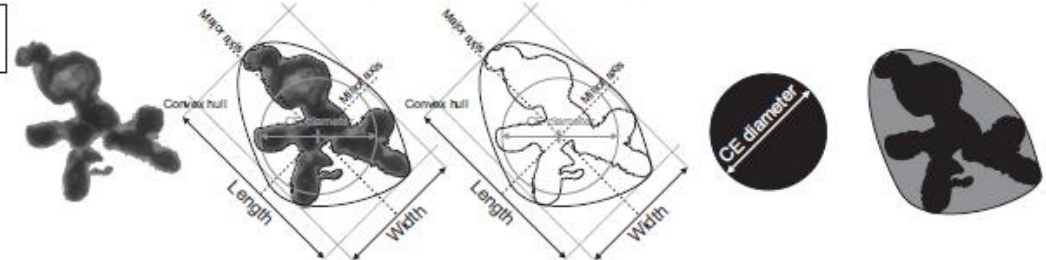

Aspect Ratio: 0.817

CE Diameter: $61.53 \mu \mathrm{m}$

Circularity: 0.785

Convexity: 0.816

Elongation: 0.183

SE Volume: $121981.44 \mu \mathrm{m}^{3}$

Solidity: 0.974

Aspect Ratio: 0.621

CE Diameter: $59.89 \mu \mathrm{m}$

Circularity: 0.667

Convexity: 0.752

Elongation: 0.379

SE Volume: $112467.58 \mu \mathrm{m}^{3}$

Solidity: 0.922

Aspect Ratio: 0.416

CE Diameter: $48.34 \mu \mathrm{m}$

Circularity: 0.678

Convexity: 0.850

Elongation: 0.584

SE Volume: $62885.91 \mu \mathrm{m}^{3}$

Solidity: 0.932

Aspect Ratio: 0.658

CE Diameter: $53.74 \mu \mathrm{m}$

Circularity: 0.341

Convexity: 0.483

Elongation: 0.342

SE Volume: $81266.48 \mu \mathrm{m}^{3}$

Solidity: 0.608

Aspect Ratio $=$ Width/Length
CE Diameter: diameter of a circle with the same area as the projected $2 \mathrm{D}$ particle image

CE Diameter: diameter of a circle with the

Circularity $=(2 \times \pi$ )
Convexity $=$ Perimeater

Elongation $=1-($ Width $/$ Length $)=1-$ Aspect Ratio

SE Volume: volume of a sphere with the same CE Diameter as the projected 2D particle image Solidity $=$ Aree comeana $/$ Area

Figure 2. Schematic illustration of major grain size and shape parameters of irregular mineral hull). 


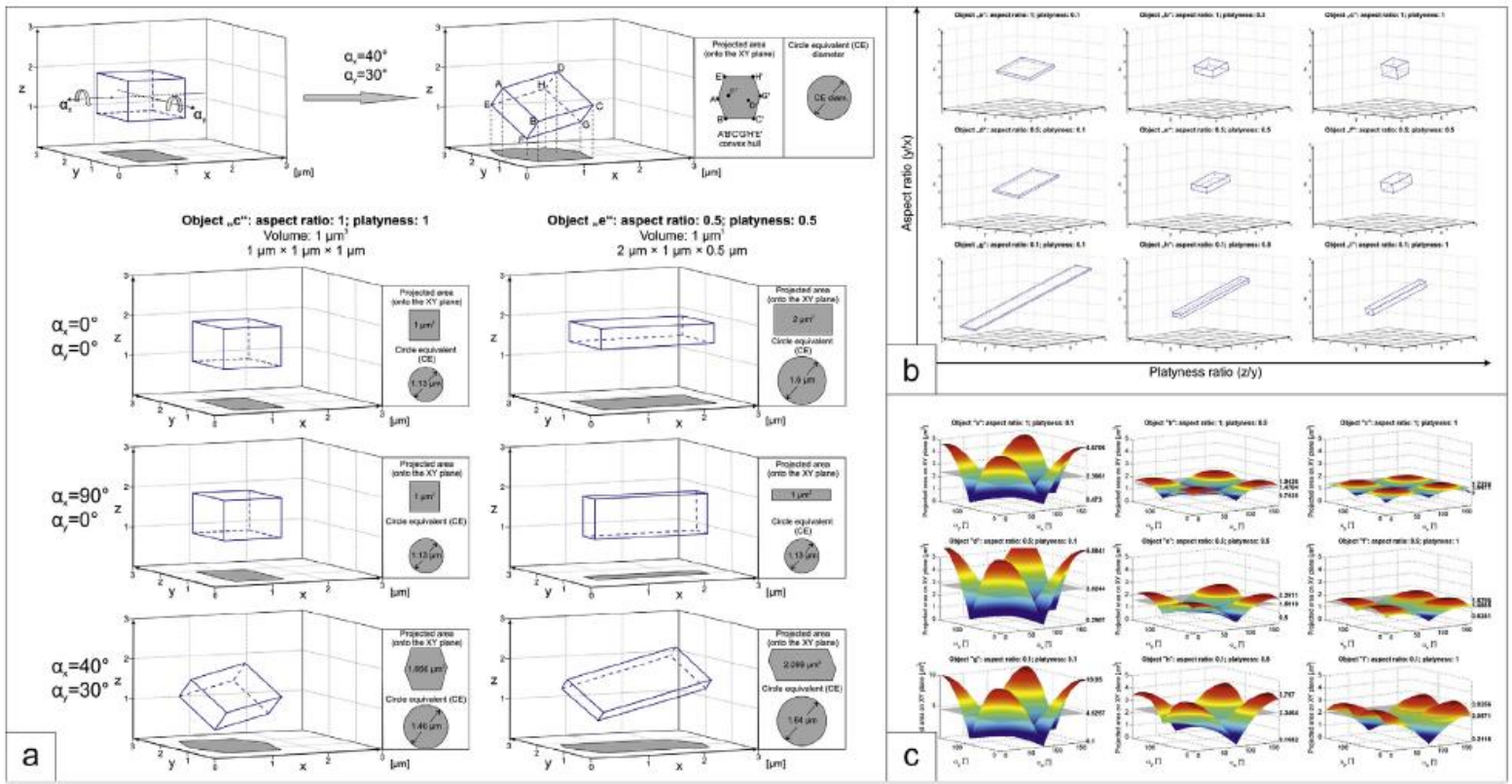

689 Figure 3. Assessment of projected area of a randomly oriented geometric objects: (a) general

690 problem of shape and rotation determined projected area; (b) presented nine simple geometric 691 objects; (c) rotation averaged projected areas as a function of rotation angles.

692 

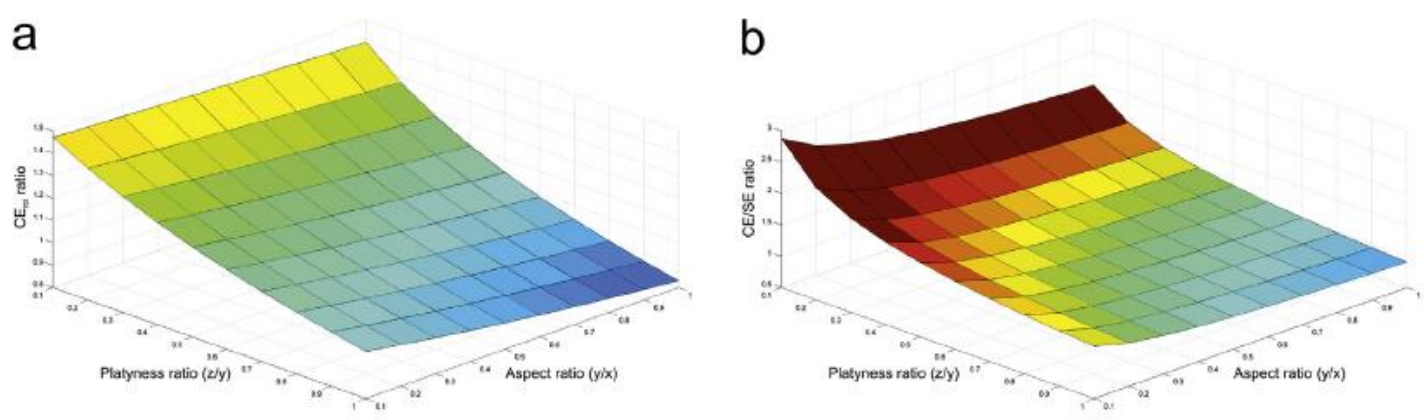

693

694 Figure 4. The introduced (a) $\mathrm{CE}_{\mathrm{rot}}$ and (b) $\mathrm{CE} / \mathrm{SE}$ ratios as a function of aspect and platyness

695 ratios of simple objects.

696 

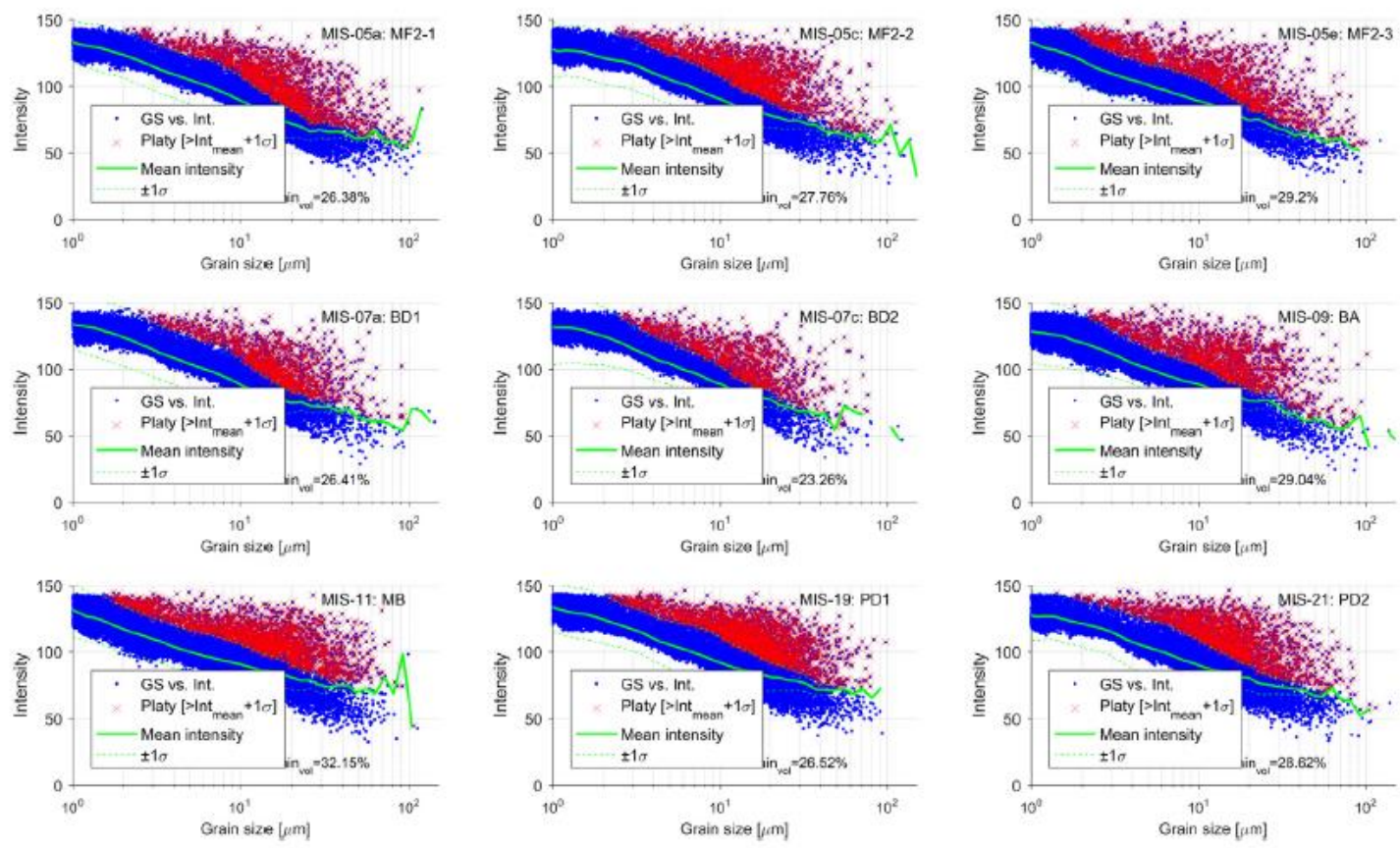

697

698 Figure 5. Assessment of amount of platy mineral particles from the grain size vs. grayscale 699 intensity scatter plots. 

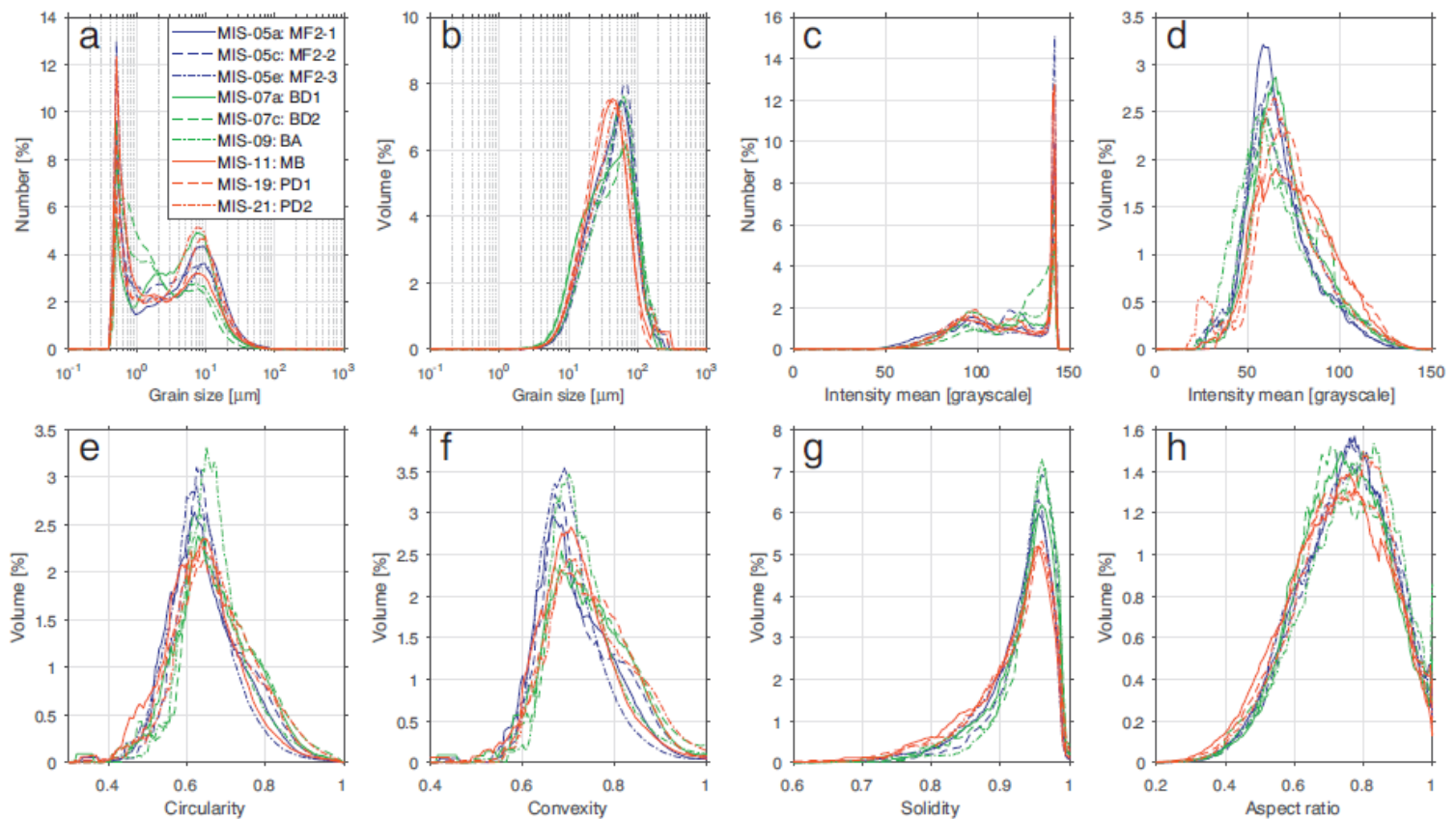

Figure 6. Grain size and shape parameter distributions of the investigated samples (a: numberbased grain size distribution; b: volume-based grain size distribution; c: number-based grayscale intensity distribution; d: volume-based grayscale intensity distribution; e: volumebased circularity distribution; f: volume-based convexity distribution; g: volume-based solidity distribution; h: volume-based aspect ratio distribution). 

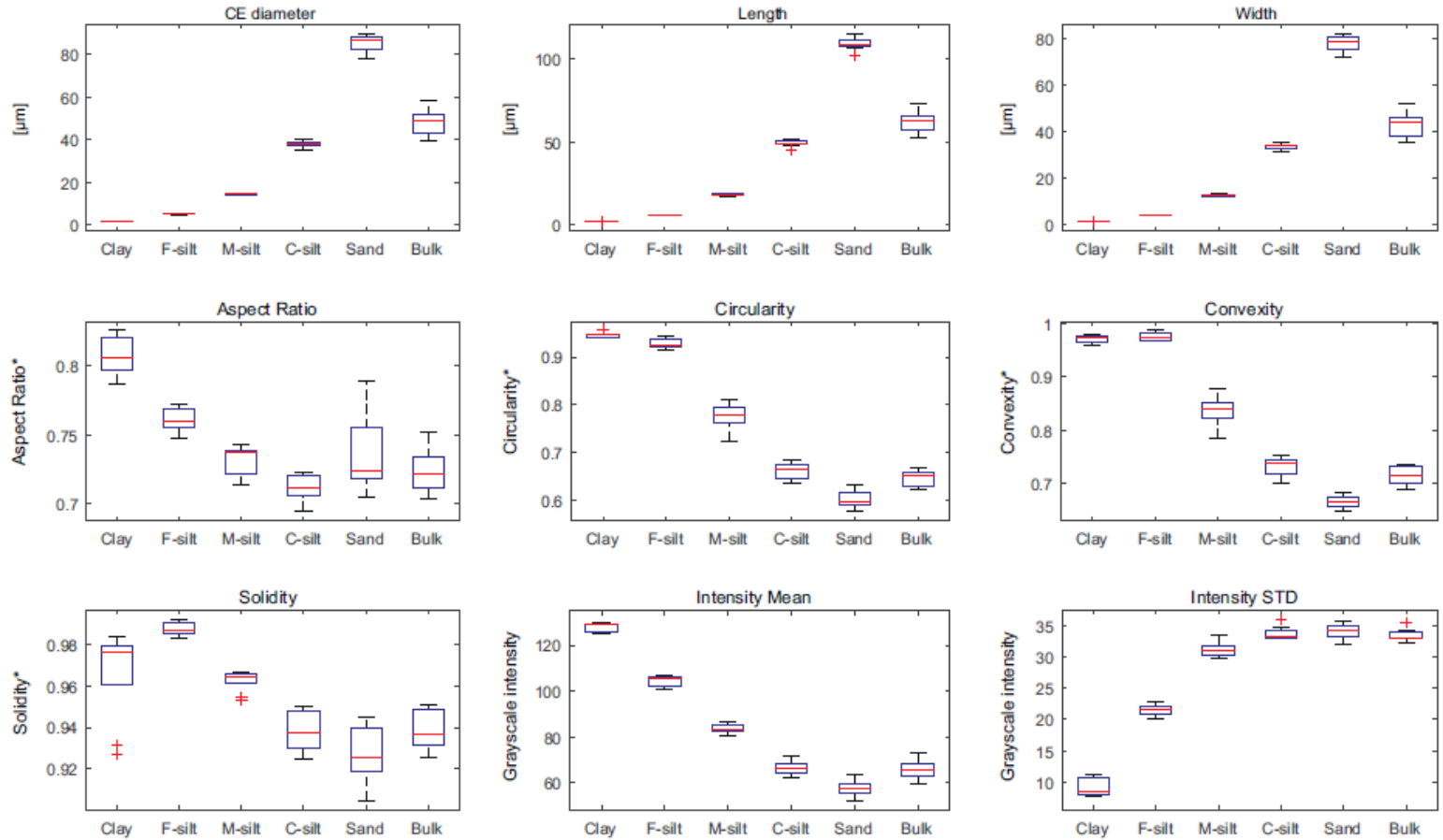

Figure 7. Box-plots of various granulometric parameters by size fractions (CE diameter: circle-

710 equivalent diameter; clay: $<2.00 \mu \mathrm{m}$; F-silt [fine silt]: 2.00-6.25 $\mu \mathrm{m}$; M-silt [medium silt]: 6.25-

$71120.00 \mu \mathrm{m}$; C-silt [coarse silt] 20.00-62.50 $\mu \mathrm{m}$; sand: larger than $62.5 \mu \mathrm{m}$ ). 


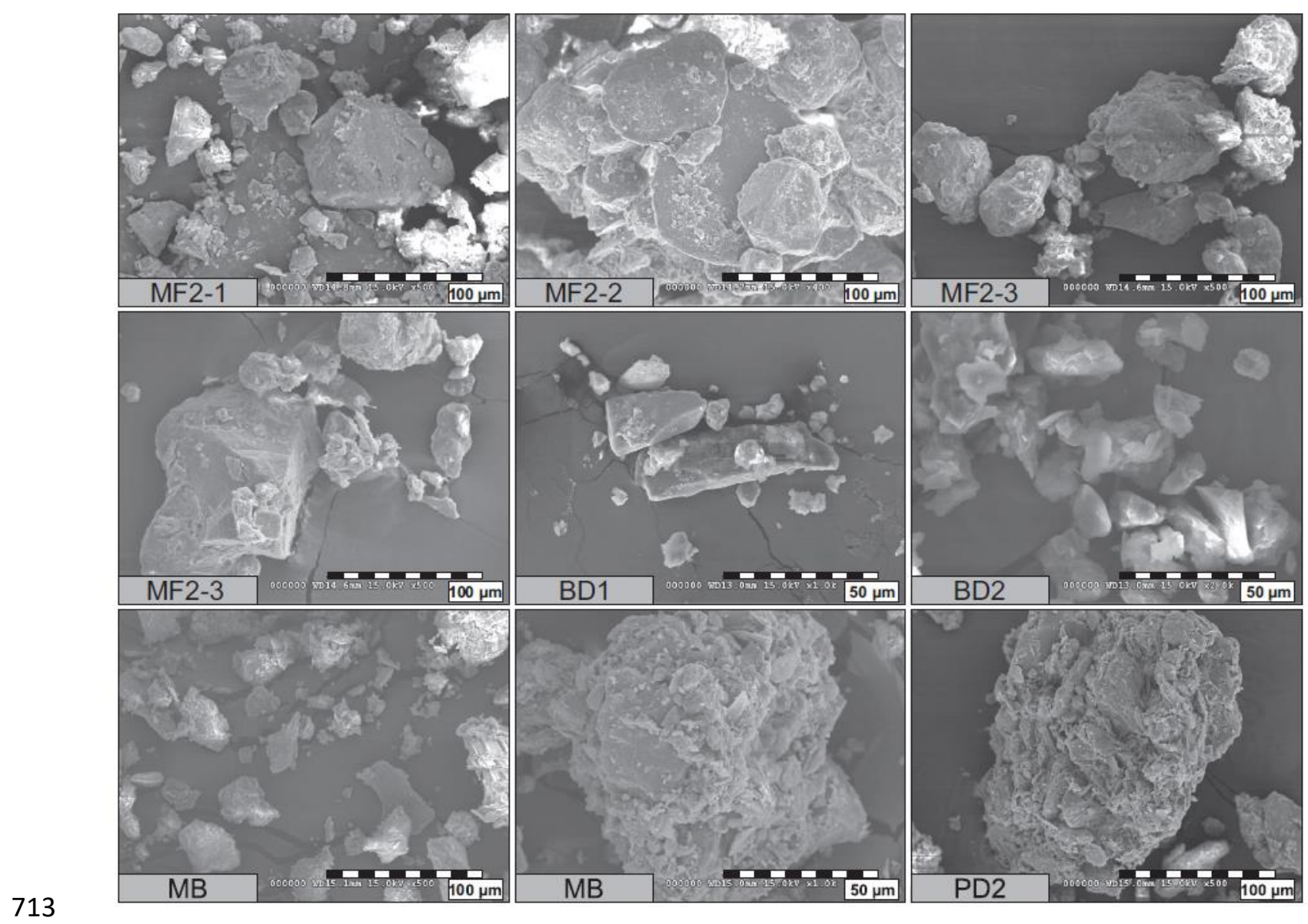

714 Figure 8. Scanning electron micrographs of paleosol samples. 

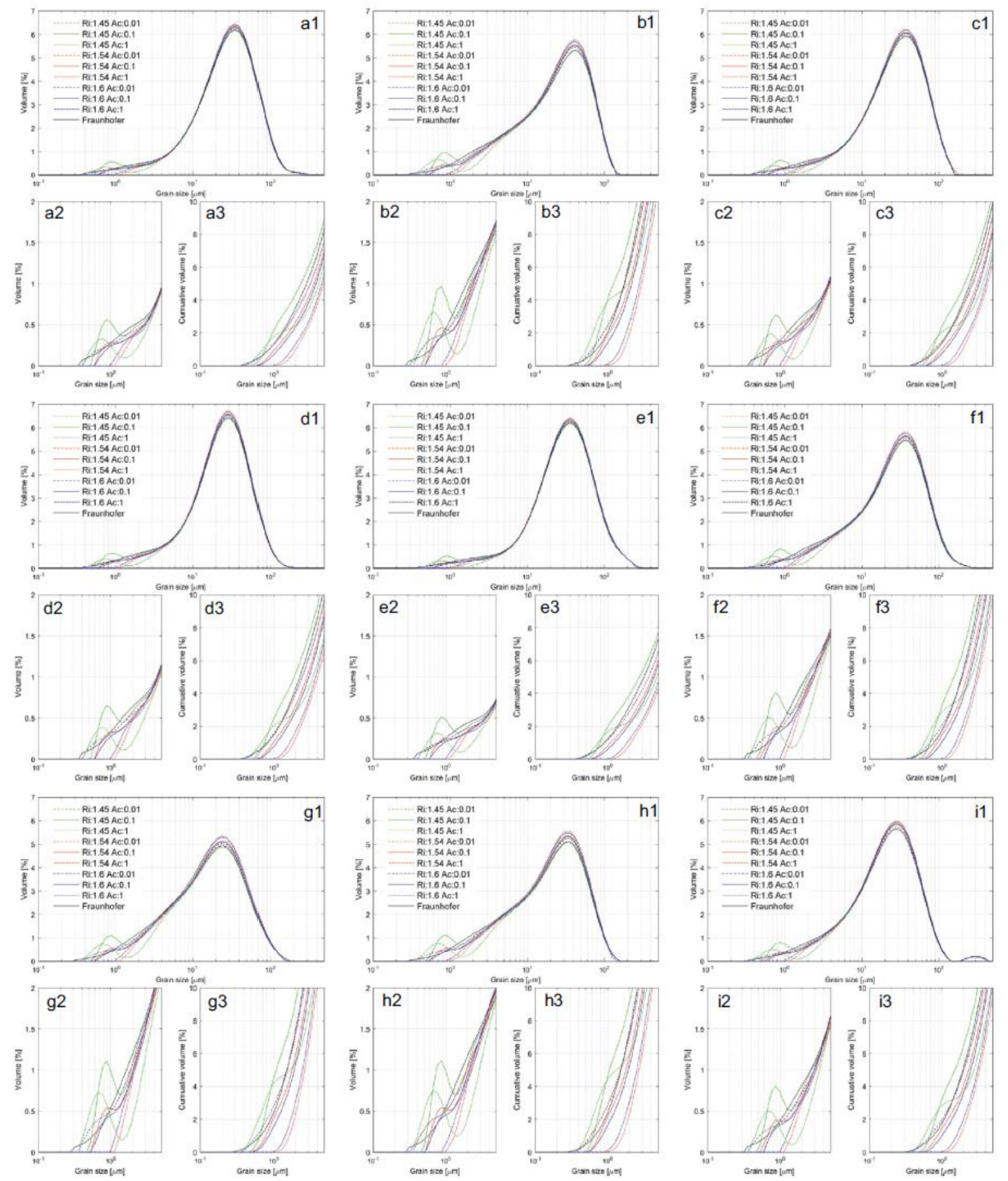

Figure 9. Laser diffraction grain size distributions of paleosol samples by using different

718 complex refractive indices (a-i refer to investigated samples [a: MF2-1; b: MF2-2; c: MF2-3;

719 d: BD-1; e: BD-2; f: BA; g: MB; h: PD1; i: PD2]; while numbers indicate the distribution types:

720 1: grain size distribution of the whole size spectrum [0.1-500 $\mu \mathrm{m}] ; 2$ : grain size distribution of

721 fine-grained fractions $[0.1-50 \mu \mathrm{m}]$; 3: cumulative grain size distribution of the fine-grained

722 fractions $[0.1-50 \mu \mathrm{m}])$. 

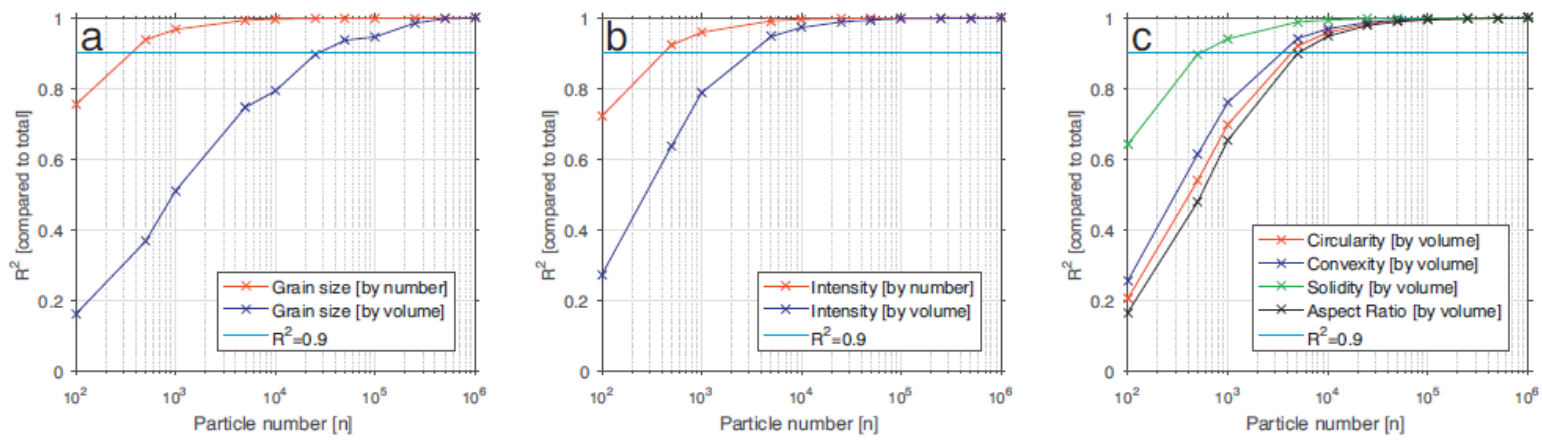

Figure 10. Relationship between number of scanned particles and representativeness of various

725 granulometric parameters (a: number- and volume-based grain size; b: number- and volumebased grayscale intensity; c: volume-based circularity, convexity, solidity and aspect ratio) for

727 the whole samples. 

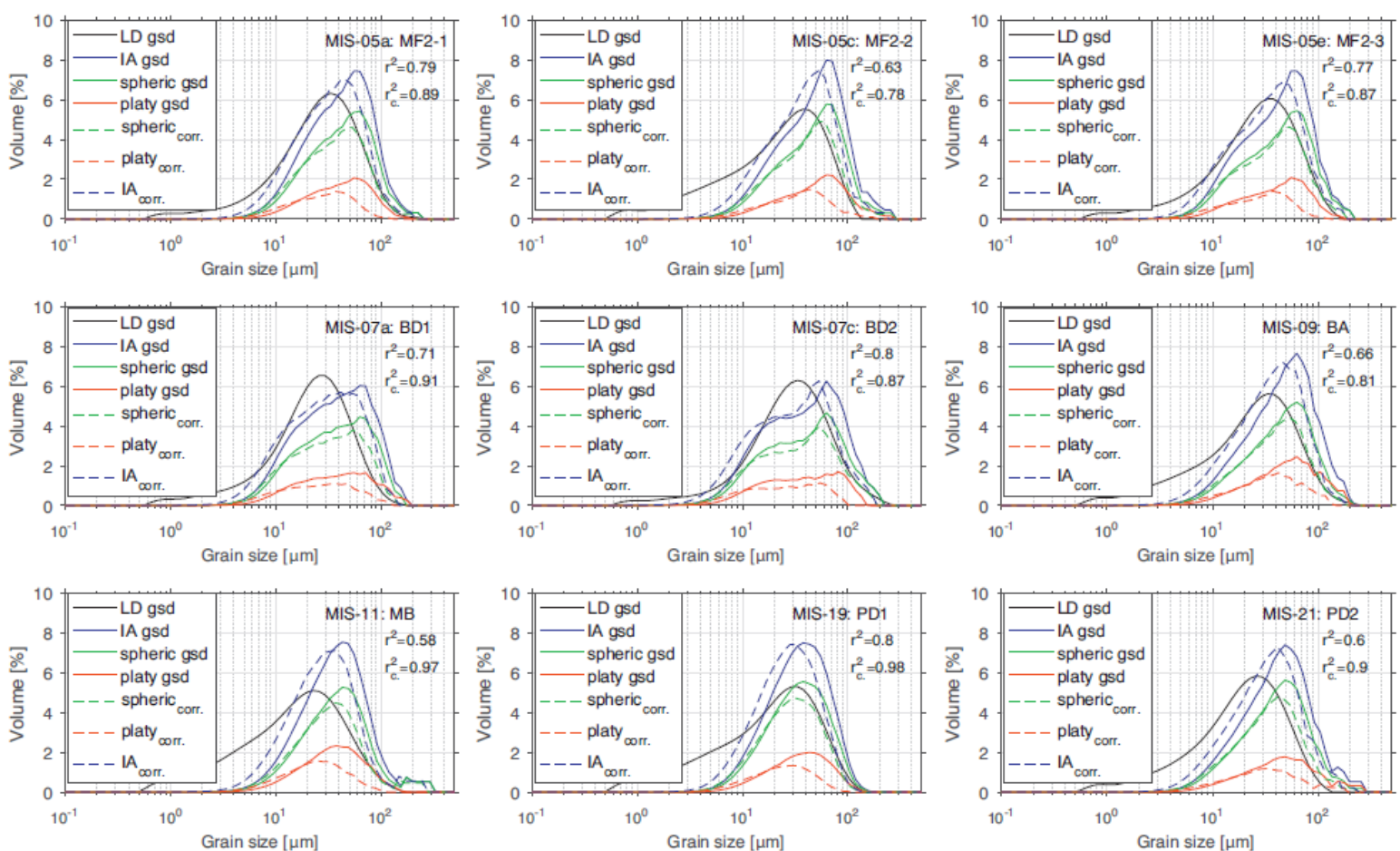

Figure 11. Grain size distributions of laser diffraction, image analysis and corrected image

731 analysis measurements (LD gsd: laser diffraction grain size distribution; IA gsd: image analysis

732 grain size distribution; 'corr' subscripts refer to corrected values). 
735 Table 1. General size and shape characteristics of the presented geometric objects and derived 736 correction factors.

\begin{tabular}{|c|c|c|c|c|c|c|c|c|c|c|c|c|c|c|c|c|c|c|c|}
\hline \multirow[t]{3}{*}{ ID } & \multirow{2}{*}{\multicolumn{2}{|c|}{ Shape parameters }} & \multirow{2}{*}{\multicolumn{3}{|c|}{ Edge lengths }} & \multirow{2}{*}{\multicolumn{2}{|c|}{ Volume }} & \multirow{2}{*}{\multicolumn{2}{|c|}{ Largest face }} & \multicolumn{8}{|c|}{ Projected areas on $\mathrm{XY}$ plane } & \multirow[t]{3}{*}{$\mathrm{CE}_{\mathrm{rot}}$ ratio $^{\mathrm{c}}$} & \multirow[t]{3}{*}{ CE/SE ratio ${ }^{d}$} \\
\hline & & & & & & & & & & \multicolumn{4}{|c|}{ Areas } & \multicolumn{4}{|c|}{ CE diameters } & & \\
\hline & Aspect ratio $(y / x)$ & $\begin{array}{l}\text { Platyness } \\
(\mathrm{z} / \mathrm{y})\end{array}$ & $\mathrm{x}$ & $\mathrm{y}$ & $\mathrm{z}$ & {$\left[\mu \mathrm{m}^{\mathrm{c}}\right]$} & $\begin{array}{l}\mathrm{SE}^{\mathrm{a}} \\
\text { diameter }\end{array}$ & Area & $\begin{array}{l}\mathrm{CE}^{\mathrm{b}} \\
\text { diameter }\end{array}$ & $\min$ & $\max$ & mean & std & $\min$ & $\max$ & mean & std & & \\
\hline a & 1 & 0.1 & 2.15 & 2.15 & 0.22 & 1 & 1.24 & 4.64 & 2.43 & 0.47 & 4.67 & 2.37 & 1.26 & 0.78 & 2.44 & 1.74 & 0.48 & 1.40 & 1.96 \\
\hline b & 1 & 0.5 & 126 & 1.26 & 0.63 & 1 & 1.24 & 1.59 & 1.42 & 0.79 & 1.94 & 1.47 & 0.32 & 1.01 & 1.57 & 1.37 & 0.16 & 1.04 & 1.15 \\
\hline c & 1 & 1 & 1.00 & 1.00 & 1.00 & 1 & 1.24 & 1.00 & 1.13 & 1.00 & 1.72 & 1.45 & 0.18 & 1.13 & 1.48 & 1.36 & 0.09 & 0.83 & 0.91 \\
\hline d & 0.5 & 0.1 & 3.42 & 1.71 & 0.17 & 1 & 1.24 & 5.85 & 2.73 & 0.29 & 5.88 & 2.82 & 1.65 & 0.61 & 2.74 & 1.90 & 0.59 & 1.44 & 2.20 \\
\hline e & 0.5 & 0.5 & 2.00 & 1.00 & 0.50 & 1 & 1.24 & 2.00 & 1.60 & 0.50 & 2.29 & 1.59 & 0.51 & 0.80 & 1.71 & 1.42 & 0.25 & 1.12 & 1.29 \\
\hline f & 0.5 & 1 & 1.59 & 0.79 & 0.79 & 1 & 1.24 & 1.26 & 1.27 & 0.62 & 1.88 & 1.49 & 0.34 & 0.89 & 1.55 & 1.38 & 0.17 & 0.92 & 1.02 \\
\hline $\mathrm{g}$ & 0.1 & 0.1 & 10.00 & 1.00 & 0.10 & 1 & 1.24 & 10.00 & 3.57 & 0.10 & 10.05 & 4.63 & 2.90 & 0.36 & 3.58 & 2.43 & 0.84 & 1.47 & 2.88 \\
\hline h & 0.1 & 0.5 & 5.85 & 0.58 & 0.29 & 1 & 1.24 & 3.42 & 2.09 & 0.17 & 3.80 & 2.35 & 1.04 & 0.46 & 220 & 1.73 & 0.44 & 121 & 1.68 \\
\hline i & 0.1 & 1 & 4.64 & 0.46 & 0.46 & 1 & 1.24 & 2.15 & 1.66 & 0.21 & 3.03 & 2.09 & 0.80 & 0.52 & 1.96 & 1.63 & 0.37 & 1.02 & 1.34 \\
\hline
\end{tabular}

737

a SE: sphere-equivalent.

c CErot ratio: ratio of the largest face area-based CE diameter.

d CE/SE ratio: ratio of SE and CE diameters. 
740 Table 2. Mean grain size and shape parameters of the investigated samples.

741

\begin{tabular}{|c|c|c|c|c|c|c|c|c|c|c|c|c|c|c|}
\hline Sample Name & CE diam. $[\mu \mathrm{m}]$ & Clay [vol.:\%] & Fine silt [vol:\%] & Medium silt [vol...] & Coarse silt [vol.\%] & Sand [vol.\%] & Length $[\mu \mathrm{m}]$ & Width $[\mu \mathrm{m}]$ & Aspect Ratio & Gircularity & Convexity & Solidity & Intensity Mean & Intensity STD \\
\hline MIS-05a [MF2-1] & 49.83 & 0.01 & 0.31 & 12.46 & 49.33 & 37.90 & 64.19 & 44.43 & 0.73 & 0.63 & 0.69 & 0.94 & 62.09 & 35.47 \\
\hline MIS-05c [MF2-2] & 58.19 & 0.01 & 0.36 & 10.77 & 42.52 & 46.34 & 72.80 & 52.01 & 0.74 & 0.64 & 0.70 & 0.95 & 63.53 & 32.97 \\
\hline MIS-05e [MF2-3] & 50.40 & 0.02 & 0.42 & 15.20 & 45.35 & 39.02 & 64,46 & 45.03 & 0.72 & 0.62 & 0.69 & 0.94 & 63.14 & 33.81 \\
\hline MIS-07a [BD1] & 42.91 & 0.02 & 0.75 & 19.81 & 45.18 & 34.25 & 5620 & 38.77 & 0.72 & 0.65 & 0.73 & 0.94 & 66.53 & 34.08 \\
\hline MIS-07c [BD2] & 45.68 & 0.06 & 1.02 & 20.38 & 40.66 & 37.88 & 57.05 & 40.48 & 0.71 & 0.67 & 0.74 & 0.95 & 65.64 & 32.94 \\
\hline MIS-11 [MB] & 42.57 & 0.02 & 0.48 & 14.57 & 57.25 & 27.68 & 56.96 & 38.03 & 0.70 & 0.63 & 0.71 & 0.93 & 72.81 & 32.82 \\
\hline MIS-19 [PD1] & 39.75 & 0.01 & 0.37 & 15.91 & 60.09 & 23.61 & 52.26 & 35.71 & 0.71 & 0.65 & 0.73 & 0.93 & 73.17 & 32.95 \\
\hline MIS-21 [PD2] & 49.09 & 0.01 & 0.46 & 13.75 & 50.81 & 34.98 & 63.07 & 44.38 & 0.72 & 0.66 & 0.73 & 0.93 & 66.57 & 32.99 \\
\hline Mean & 47.35 & 0.01 & 0.41 & 13.40 & 51.42 & 34.76 & 61.47 & 42.48 & 0.72 & 0.64 & 0.71 & 0.94 & 66.65 & 33.22 \\
\hline STD & 6.02 & 0.02 & 023 & 3.36 & 6.58 & 7.02 & 6.77 & 5.29 & 0.02 & 0.02 & 0.02 & 0.01 & 4.59 & 0.96 \\
\hline
\end{tabular}

742 
743 Table 3. Mean values of various granulometric parameters by size fractions.

\begin{tabular}{|c|c|c|c|c|c|c|c|c|c|}
\hline Fractions & CE diam. $[\mu \mathrm{m}]$ & Length $[\mu \mathrm{m}]$ & Width $[\mu \mathrm{m}]$ & Aspect Ratio & Gircularity & Convexity & Solidity & Intensity Mean & Intensity STD \\
\hline Clay $(<2 \mu \mathrm{m})$ & 1.51 & 1.84 & 1.44 & 0.80 & 0.95 & 0.97 & 0.97 & 127.00 & 9.80 \\
\hline Medium silt $(6.25-20 \mu \mathrm{m})$ & 14.78 & 18.39 & 12,89 & 0.73 & 0.77 & 0.84 & 0.96 & 84.00 & 30.86 \\
\hline Bulk & 47.35 & 61.47 & 42.48 & 0.72 & 0.64 & 0.71 & 0.94 & 66.65 & 33.22 \\
\hline
\end{tabular}

744

745 
747 Table 4. Shape parameters of 30-120 $\mu \mathrm{m}$ quartz, feldspar and bulk samples.

\begin{tabular}{lllllll}
\hline $\begin{array}{l}\text { Samples } \\
{[30-120 \mu \mathrm{m}]}\end{array}$ & $\begin{array}{l}\text { Aspect } \\
\text { Ratio }\end{array}$ & Circularity & Convexity & Solidity & $\begin{array}{l}\text { Intensity } \\
\text { mean }\end{array}$ & $\begin{array}{l}\text { Intensity } \\
\text { STD }\end{array}$ \\
\hline Quartz & 0.7361 & 0.6133 & 0.6762 & 0.9284 & 60.3 & 33.29 \\
Feldspar & 0.7164 & 0.6136 & 0.6846 & 0.929 & 56.57 & 33.61 \\
Bulk & 0.7208 & 0.6245 & 0.6922 & 0.929 & 62.24 & 33.57 \\
\hline
\end{tabular}

748

749 